\title{
A catalogue of spatially resolved kinematics of galaxies: Bibliography ${ }^{\star}$
}

\author{
Ph. Prugniel ${ }^{1}$, A. Zasov $^{2}$, G. Busarello ${ }^{3}$, and F. Simien ${ }^{1}$ \\ 1 CRAL-Observatoire de Lyon, CNRS: UMR 142, F-69561 St-Genis-Laval Cedex, France \\ 2 Sternberg Astronomical Institute, Universitetskij prospect, 119899, Moscow, Russia \\ 3 Osservatorio Astronomico di Capodimonte, via Moiariello 16, I-80131 Napoli, Italy
}

Received April 24; accepted April 28, 1997

\begin{abstract}
We present a catalogue of galaxies for which spatially resolved data on their internal kinematics have been published; there is no a priori restriction regarding their morphological type. The catalogue lists the references to the articles where the data are published, as well as a coded description of these data: observed emission or absorption lines, velocity or velocity dispersion, radial profile or $2 \mathrm{D}$ field, position angle.
\end{abstract}

Key words: catalogs - galaxies general — galaxies: kinematics and dynamics

\section{Introduction}

Data on the internal kinematics of galaxies are either spatially unresolved, like central or mean velocity dispersion or like HI velocity profile or width, or may be resolved. Catalogues of unresolved kinematics exist. Huchtmeier \& Richter (1989) and Paturel et al. (1990; see also Bottinelli et al. 1990) presented compilations of HI width measurements. McElroy (1995) or Prugniel \& Simien (1996: hereafter PS96) give central velocity dispersions for the stellar component. A compilation of spatially resolved kinematics has been published by Corradi \& Capaccioli (1991), but it is limited to a sample of "normal" spiral galaxies. Busarello et al. (1989) published an atlas of stellar kinematics of early-type galaxies. Finally, a catalogue of maximum rotational velocity of galaxies (stellar kinematics) is published in PS96.

The goal of the present project is to build a catalogue gathering references to all spatially resolved studies,

Send offprint requests to: Ph. Prugniel (prugniel@obs.univlyon1.fr)

* Tables 1, 2, and 3 are proposed in electronic form only, and are available from the CDS, via anonymous ftp to cdsarc.u-strasbg.fr (to 130.79.128.5) or via http://cdsweb.ustrasbg.fr/Abstract.html for all galaxies, whatever the gas or stellar component is concerned. The catalogue consists in a list of galaxies for which references to the original papers are given together with a short coded description of the data.

The catalogue has been assembled from merging the lists of Corradi \& Capaccioli (1991) and PS96). A bibliographical list distributed on the WEB by Demin et al. (1997) has also been used, and we scanned the literature to add new references. In total, the catalogue contains 4536 observations of 2207 galaxies referring to 777 published papers. The largest source is Mathewson et al. (1992) $(\mathrm{H} \alpha$ velocity profiles of 970 galaxies) representing about $20 \%$ of the whole catalogue. The distributions of the number of references and of measurements are shown in Fig. 1 as a function of the year of publication.

\section{Description of the catalogue}

The catalogue consists in three files:

- (1) the list of observations (Table 1),

- (2) the bibliography (Table 2),

- (3) the coding of the spectral lines (Table 3).

The list of observations consists of records containing:

- (i) the identification of the galaxy,

- (ii) the code of the reference,

- (ii) a coded description of the observation.

The identifiers of the galaxies follow the scheme used in PS96, they are also valid Simbad identifiers. In addition, the coordinates are given. The code adopted for the reference is the BIBCODE defined by the CDS and also adopted by NED or ADS.

The description of an observation closely follows that defined in Corradi \& Capaccioli (1991). The first four characters of this code indicate:

- (i) the domain of observation (O: optical, $\mathrm{R}$ : radio),

- (ii) the type of spectral feature (E: emission; A: absorption), 


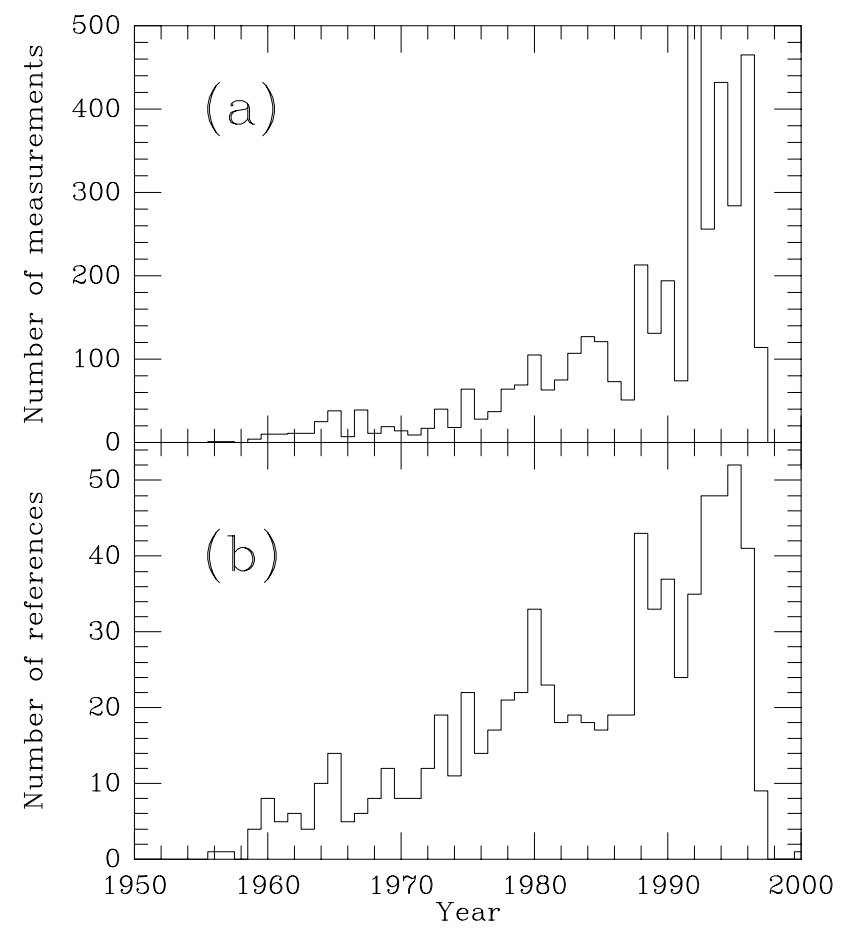

Fig. 1. Content of the catalogue. Distributions of the number of measurements a) and of the number of references $\mathbf{b}$ ) compiled in the catalogue as a function of the year of publication. References published before 1950 are not seen, and the peak corresponding to the 970 galaxies measured by Mathewson et al. (1992) is truncated

- (iii) the range of data $(1: 1 \mathrm{D}$, e.g. rotation curve; 2 : 2D, e.g. velocity field),

- (iv) the type of data (V: velocity; D: dispersion; P: position-velocity diagram).

Then, when available in the original source, the observed position angles are indicated. They are coded in two fields, as follows:

- (i) observed axis (MJ: major axis; MI: minor axis; BA: along the bar; PA: pair axis, for binary or doublenucleed galaxies),

- (ii) position angle in degrees, counted as usual from North though East.
It is followed by the list of spectral lines (or features) used, coded as described in Table 3.

\section{Conclusion}

The bibliographic catalogue is a first step which we expect to continue with a catalogue gathering the kinematical profiles themselves. For the recent literature, these are often available in electronic form, and for older reference in the form of printed tables. Unfortunately, many data are only presented in graphical form and we will probably not be able to digitize them.

This catalogue is also connected to an hypertext interface to databases and catalogues, HYPERCAT, currently under development at the Observatoire de Lyon (http://www-obs.univ-lyon1.fr/ $\sim$ prugniel/cgi-bin/hypercat/).

Acknowledgements. We acknowledge the use of SIMBAD (operated at Observatoire de Strasbourg, http://cdsweb.ustrasbg.fr), LEDA (Observatoire de Lyon, http://leda.univlyon1.fr/) and of the ADS (Nasa, mirrored at CDS, http://cdsads.u-strasbg.fr/).

\section{References}

Bottinelli L., Gouguenheim L., Fouqué P., Paturel G., 1990, A\&AS 82, 391

Busarello G., Longo G., Di Martino F., 1989, Atlas of Velocity Dispersion Profiles and Rotation Curves for Elliptical and Lenticular Galaxies, Liguori Napoli

Corradi R., Capaccioli M., 1991, A\&AS 90, 121

Demin V.V., Zasov A.V., Petrochenko L.N., 1997, http://www.sai.msu.su/groups/galaxies/catalog.html

Huchtmeier W.K., Richter O.G., 1989, A General Catalogue of HI Observations of Galaxies. Springer-Verlag

Mathewson D.S., Ford V.L., Buchhorn M., 1992, ApJS 81, 413

McElroy D., 1995, ApJS 100, 105

Paturel G., Bottinelli L., Gouguenheim L., Fouqué P., 1990, Observatoires de Lyon et Paris-Meudon, Monographies de la base de données, 2

Prugniel Ph., Simien F., 1996, A\&A 309, 749 